\title{
COMPENSATORY OVARIAN HYPERTROPHY CAN BE OBTAINED IN NEONATAL RATS
}

\author{
JANIS L. DUNLAP AND A. A. GERALL \\ Department of Psychology, Tulane University, New Orleans, \\ Louisiana 70118, U.S.A.
}

(Received 14th August 1972)

Several recent studies have disagreed as to whether compensatory ovarian hypertrophy $(\mathrm{COH})$ occurs in rats younger than 20 days of age. In female rats hemiovariectomized at 10 days of age by Ojeda \& Ramírez (1972), there was no significant increase in the weight of the remaining left ovary at 12,15 and 20 days of age when compared to that in animals subjected to sham operation. Baker \& Kragt (1969) reported that excising the right ovary at birth did not cause the remaining ovary to be significantly heavier than the left ovary in control rats at 10,15 and 20 days of age. By contrast, Gerall \& Dunlap (1971) and Dunlap, Preis \& Gerall (1972) have reported significant $\mathrm{COH}$ in the right ovary within 10 days in rats hemispayed at birth. Negative results have been interpreted as indicating either that the neonatal female's gonad does not secrete oestrogen or that its hypothalamic-hypophysial system is not responsive to circulating hormones. Since these results are relevant to understanding the neonatal female's neuroendocrine system, it is important to discern the basis of the discrepancy between these studies and whether, in fact, $\mathrm{COH}$ occurs in the female rat before 20 days of age.

It is contended that $\mathrm{COH}$ is a reliable phenomenon in the neonatal rat and that the major difference between studies, so far as can be ascertained, is based on whether extraneous tissue is removed from the ovaries before weighing them. Including oviduct, fat and connective tissue with the ovary not only produces incorrect large weights but increases the variability of weights across subjects. This variability should decrease the probability that small amounts of $\mathrm{COH}$ will be detected statistically. In the following experiment, weights of cleaned and non-cleaned ovaries were compared to determine whether excess tissue could account for the difference in absolute ovarian weights and lack of $\mathrm{COH}$ reported by several investigators.

Sprague-Dawley females born in the Tulane Laboratory were mated and maintained in a room with a reversed 12-hr light/12-hr dark illumination cycle. Pregnant females were observed hourly on Day 22 of gestation. The day on which litters were born was designated Day 1 of life.

Twenty-eight female offspring from six litters served as subjects. On Day 1, fourteen females from these litters were hemiovariectomized and fourteen control litter mates were subjected to sham operation at the same time, using ice anaesthesia. On Day 10, the right ovary was excised from all twentyeight females and weighed immediately on a Mettler Type M5 electronic bal- 
ance with a precision of $\pm 0.001 \mathrm{mg}$ and an accuracy in the optical range of $\pm 0.002 \mathrm{mg}$. On the day of removal of the right ovary, pairs of litter mates, one member hemiovariectomized and the other subjected to sham operation, were randomly assigned to one of two groups in which ovaries were either cleaned of surrounding tissue before weighing or simply severed from the uterus. The design was a $2 \times 2$ factorial involving type of operation on Day 1 , hemiovariectomy versus sham operation, and status of ovary weighed on Day 10 , cleaned versus non-cleaned. The resulting four groups were represented in any given litter, with each litter contributing at least one female to each group.

Ovaries were cleaned while still attached to the uterus. Using microdissecting forceps and dissecting microscope, the experimenter carefully peeled off oviduct, fat and surrounding membrane. Non-cleaned ovaries were cut from the uterus with scissors with the aid of a dissecting microscope. Ovaries were placed in Bouin's solution and subsequently stained with haematoxylin and eosin. Tissue was cut at $10 \mu \mathrm{m}$ for examination.

Table 1. Right ovary weight in 10-day-old female rats

\begin{tabular}{cc|cc|cc}
\hline \multirow{2}{*}{ Pair no. } & Litter no. & \multicolumn{2}{|c|}{ Cleaned } & \multicolumn{2}{c}{ Non-cleaned } \\
\cline { 3 - 6 } & & $\begin{array}{c}\text { Hemi- } \\
\text { ovariectomy }\end{array}$ & $\begin{array}{c}\text { Sham } \\
\text { operation }\end{array}$ & $\begin{array}{c}\text { Hemi- } \\
\text { ovariectomy }\end{array}$ & $\begin{array}{c}\text { Sham } \\
\text { operation }\end{array}$ \\
\hline 1 & 1 & 0.794 & 0.540 & 1.345 & 1.008 \\
2 & 2 & 0.863 & 0.789 & 2.691 & 2.471 \\
3 & 3 & 0.881 & 0.643 & 2.400 & 2.041 \\
4 & 3 & 0.618 & 0.205 & 2.071 & 1.799 \\
5 & 4 & 0.840 & 0.601 & 2.197 & 1.367 \\
6 & 5 & 0.585 & 0.722 & 1.487 & 1.588 \\
7 & 6 & 0.619 & 0.497 & 1.278 & 1.732 \\
Mean & & 0.743 & 0.571 & 1.92 & 1.71 \\
(S.D.) & $(0.130)$ & $(0.140)$ & $(0.556)$ & $(0.469)$ \\
\multicolumn{2}{l|}{$\begin{array}{c}\text { Mean body weight (g) } \\
\text { (S.D.) }\end{array}$} & 17.4 & 18.5 & 16.9 & 18.3 \\
\multicolumn{2}{l|}{} & $(3.1)$ & $(2.9)$ & $(2.9)$ & $(3.5)$ \\
\hline
\end{tabular}

Ovary weights are expressed in $\mathrm{mg}$.

Table 1 presents the weights of cleaned and non-cleaned ovaries removed at 10 days of age from females that had been hemiovariectomized on Day 1 or subjected to sham operation. As reported previously (Gerall \& Dunlap, 1971; Dunlap et al., 1972), cleaned ovaries from 10-day-old females hemiovariectomized at birth were significantly heavier than those from paired control litter mates $(F=6.845$, d.f. $=1 / 6, P<0.05)$. However, non-cleaned ovaries of 10-day-old litter mates were not reliably heavier as a result of the Day-1 operation. They were also two to three times heavier than those cleaned before weighing. Microscopic examination of these ovaries revealed substantial and variable amounts of fat, oviduct and connective tissue. Cleaned ovaries, in contrast, had very little fat and no oviduct. Analysis of variance performed on the ratios of ovary weight/body weight yielded the same results, with hemiovariectomy producing a greater increase in weight by Day 10 in the cleaned condition only $(F=12.293$, d.f. $=1 / 6, P<0.05)$. Body weight did not differ 
significantly among the groups, though hemiovariectomized females were slightly lighter than their control litter mates.

The present study and two previous studies (Gerall \& Dunlap, 1971; Dunlap et al., 1972) have demonstrated significant $\mathrm{COH}$ by 10 days of age in female rats hemiovariectomized at birth. The most apparent difference between the studies under consideration is that the absolute weights of the neonatal ovaries reported by Baker \& Kragt (1969) and Ojeda \& Ramírez (1972) are consistently heavier than those measured by Gerall \& Dunlap (1971) and Dunlap et al. (1972). The 12-day-old mean ovary weight for control subjects reported by Ojeda \& Ramírez (1972) was $1.4 \mathrm{mg}$. The mean weight reported by Baker \& Kragt (1969) was $1.6 \mathrm{mg}$ for the 10-day-old ovary. These values are approximately equivalent to those of the present study obtained when ovaries were weighed without being cleaned of surrounding tissue and oviduct. Mean left ovary weights of 30 -day-old animals reported by these investigators were $8.9 \mathrm{mg}$ (Ojeda \& Ramírez, 1972) and approximately $9.4 \mathrm{mg}$ (Baker \& Kragt, 1969) and at 34 days, $8.7 \mathrm{mg}$ (Dunlap et al., 1972). At later ages, oviduct and extraneous tissue are probably removed by all experimenters from these larger, more developed ovaries before weighing. Thus, it is unlikely that strain differences can account for the differences in weights found at earlier ages.

Ojeda \& Ramírez (1972) weighed the remaining ovary at 2, 5 and 10 days following 10-day-old hemiovariectomy in contrast to the constant 9-day period used by Dunlap et al. (1972). The percentage of hypertrophy found by Ojeda \& Ramírez (1972) at 12 days was approximately $22 \%$ and at 20 days, $10 \%$. Dunlap et al. (1972) also reported the same trend of greater percentage COH by Day $10(42 \%)$ than by Day $19(27 \%)$, holding time since hemispaying constant. In the present study, a small mean increase in ovary weight was found in hemispayed subjects in the non-cleaned condition. The variability added by non-ovarian tissue, however, was too great to obtain statistically significant results. By cleaning ovaries, using greater precision in weighing, and controlling for litter effects, $\mathrm{COH}$ can be found in the neonatal female rat. This finding does not establish that the neonatal ovary is secretory or that the hypothalamic-hypophysial axis is differentiated to respond to ovarian feedback, but $\mathrm{COH}$ does occur, and this fact has theoretical and functional significance.

This research was supported by Research Grant HD 00867-10 from the National Institute of Child Health and Human Development, USPHS.

\section{REFERENCES}

BAKER, F. D. \& KRAGT, C. L. (1969) Maturation of the hypothalamic-pituitary-gonadal negative feedback system. Endocrinology, 85, 522.

Dunlap, J. L., Preis, L. K., Jr \& Gerall, A. A. (1972) Gompensatory ovarian hypertrophy as a function of age and neonatal androgenization. Endocrinology, 90, 1309.

Gerall, A. A. \& Dunzap, J. L. (1971) Evidence that the ovaries of the neonatal rat secrete active substances. F. Endocr. 50, 529.

OJeDA, S. R. \& Ramfrez, V. D. (1972) Plasma level of LH and FSH in maturing rats: response to hemigonadectomy. Endocrinology, 90, 466. 